Pathologe 2008 · [Suppl 2] 29:371-373

DOI 10.1007/s00292-008-1052-9

Online publiziert: 28. September 2008

(c) Springer Medizin Verlag 2008

\title{
G.B. Baretton
}

Institut für Pathologie, Universitätsklinikum Dresden

\section{Bericht der AG Gastrointestinale Pathologie}

reich gewesen. Die Detektion von tumorspezifischen Methylierungsereignissen mittels methylierungsspezifischer PCR kann in Zukunft zur Früherkennung von Neoplasien und Vorläufern, Rezidivüberwachung, Risikoeinschätzung und als prognostischer Marker verwendet werden. So wird z. B. die Methylierung des MGMTGens beim Gliom benutzt, um die Wirksamkeit alkylierender Substanzen einzuschätzen. In der molekularen Onkologie stellt die Identifizierung von Genen, die tumorspezifische CpG-Methylierung aufweisen, momentan einen Schwerpunkt dar. Hierzu wurden von der Arbeitsgruppe Hermeking zum einen Kandidatengene untersucht (wie die p53-regulierten Gene 14-3-3sigma und miR-34a). Beide Gene sind bei verschiedenen GI-Tumoren zu einem hohen Prozentsatz durch CpG-Methylierung inaktiviert. Zudem wurden genomweite Reexpressionsanalysen nach pharmakologischer Revertierung der CpG-Methylierung durch eine Kombination aus Azacytidin und Trichostatin A (einem HDAC-Inhibitor) durchgeführt. Anschließend wurden die induzierten Gene auf das Vorhandensein von methylierten CpG-Inseln in Tumoren untersucht. In Zukunft könnte die Kombination der 8 identifizierten Methylierungsmarker, die man in Körperflüssigkeiten mittels PCR nachweisen kann, bei bestimmten Tumorarten diagnostisch relevant sein.

In der Rubrik „Neues in der GI-Pathologie" boten im 2. Teil Kurzreferate einen Überblick über das aktuelle Regressionsgrading im Ösophagus und Magen (S.E. Baldus, Köln), im Kolorektum (D. Aust, Dresden) sowie im Pankreas (J. Lüttges, Saarbrücken):

Lokal fortgeschrittene Ösophagus- und Magenkarzinome haben nach wie vor eine schlechte Prognose. Aufgrund der Er- gebnisse neuerer Studien (z. B. „MAGICTrial“) stellt die neoadjuvante (Radio-) Chemotherapie derzeit den Standard der Therapie von Ösophagus- und Magenkarzinomen in den UICC-Stadien II und III dar. Jedoch besteht die Problematik, dass verlässliche Parameter für eine Prädiktion des Therapieerfolges fehlen. Zudem ist die nach klinischen Kriterien erfolgende „Responseevaluation“ ungenau. Daher stellt sich die Frage, ob ein posttherapeutisches histomorphologisches Regressionsgrading Aussagen über den Therapieerfolg zulässt. In den vergangenen Jahren wurden Responseklassifikationen für maligne Tumoren verschiedener Organe entwickelt. Bezüglich der Ösophagus- und Magenkarzinome erscheint dabei ein histomorphologisches Regressionsgrading sinnvoll, das eine Gruppe von Patienten mit „major response“ (RG III: nahezu komplette Regression, $<10 \%$ vitaler Resttumor und RG IV: komplette Regression, kein vitaler Resttumor) und eine Gruppe von Patienten mit "minor response“ (RG I: geringe/keine Regression, >50\% vitaler Resttumor und RG II: partielle Regression, $<50 \%$ und $>10 \%$ vitaler Resttumor) unterscheidet.

Dabei ist eine Unterteilung aufgrund des prozentualen vitalen Residualtumors notwendig. In einem Kölner Patientenkollektiv wiesen dabei sowohl Plattenals auch Adenokarzinome des Ösophagus mit „major response“ nach Radiochemotherapie und Ro-Resektion eine signifikant höhere Überlebenswahrscheinlichkeit als solche mit „minor response“ auf. Dieser Effekt war in der Langzeitbeobachtung bei Adenokarzinomen besonders ausgeprägt. Darüber hinaus erwies sich der Lymphknotenstatus (ypN) als ein weiterer prognostisch unabhängiger relevanter Parameter. Auch beim Magen- 
karzinom stellt aufgrund der verfügbaren Daten ein vitaler residualer Tumor von weniger als $10 \%$ einen positiven prognostischen Faktor dar.

Ähnlich wie für die lokal fortgeschrittenen Ösophagus- und Magenkarzinome gilt auch für das lokal fortgeschrittene Rektumkarzinom die neoadjuvante Therapie, in aller Regel als Radiochemotherapie durchgeführt, insbesondere nach den Ergebnissen der deutschen Rektumstudie als Goldstandard. Mithilfe der neoadjuvanten Radiochemotherapie kann insbesondere das Lokalrezidivrisiko des Rektumkarzinoms erheblich gesenkt und damit die Lebensqualität der Patienten deutlich verbessert werden. Das histologische Regressionsgrading stellt einen Surrogatmarker für die Effektivität der neoadjuvanten Therapie dar, mit dessen Hilfe möglicherweise das Risiko eines Rezidivs und die evtl. Notwendigkeit einer zusätzlichen (aggressiveren) adjuvanten Therapie vorausgesagt werden können. Das Regressionsgrading zeigt zudem Einfluss auf das krankheitsfreie Überleben der Patienten. Für das Rektumkarzinom hat sich das Regressionsgrading nach Dworak (1994) etabliert, dessen Anwendung auch in den neuen Leitlinien zum kolorektalen Karzinom (2008) empfohlen wird. Dworak unterscheidet insgesamt 5 Regressionsgrade: Grad o zeigt weder Fibrose/Nekrose noch zelluläre bzw. strukturelle Veränderungen (keine Regression), Grad 1 eine dominante Tumormasse mit deutlicher Fibrose und/ oder Vaskulopathie, Grad 2 eine dominante Fibrose mit wenigen Tumorzellresiduen, die jedoch in der Übersicht bereits gut erkennbar sind, Grad 3 ebenfalls eine dominante Fibrose mit nur noch einzelnen, in der Übersicht kaum noch erkennbaren Tumorzellresiduen, und Grad 4 zeigt schließlich die komplette Regression ohne Nachweis von Tumorzellresiduen. Essenzielle Voraussetzung für eine zuverlässige Bestimmung des Tumorregressionsgrads ist die sorgfältige Aufarbeitung des Präparates, wobei hier prinzipiell die Regel gilt: Je weniger Veränderungen makroskopisch erkennbar sind, umso mehr Gewebe muss histologisch untersucht werden. Als Richtlinie gelten mindestens 4 Kapseln aus dem Tumor, die die Ausdehnung des Tumors umfassen sollten. Bei fehlendem makroskopischem Nachweis eines Tumors sollte das auffällige Schleimhautareal (z. B. Ulkus) komplett eingebettet werden. Sind auf den ersten Schnittstufen keine Tumorzellen nachweisbar, sollte das Material in weiteren Schnittstufen aufgearbeitet werden. Zur Abgrenzung von residuellen Tumorzellen, insbesondere in Schleimseen, erweist sich eine immunhistologische Reaktion gegen Panzytokeratine als hilfreich.

Das duktale Pankreaskarzinom weist unter den 6o häufigsten Malignomen nach wie vor die schlechteste Prognose auf, weswegen multimodale Therapiekonzepte auch unter Einbeziehung adjuvanter oder neoadjuvanter Radiochemotherapie derzeit diskutiert werden. Die „großen adjuvanten Studien“ (GITSG, EORTC, ESPAC 1) der Vergangenheit zeigten mit $z$. T. geringen Fallzahlen und einem schwierigen, nicht unwidersprochenen Studiendesign durchweg eine signifikante geringe Verbesserung der medianen Überlebenszeit. Eine überzeugende Verbesserung des medianen rezidivfreien Intervalls (10,4 vs. 24,8 Monate) und des medianen Überlebens (20,2 vs. 22,2 Monate erzielt die CONKO-oo1-Studie (Oettle et al. JAMA 2007). Da jedoch aufgrund perioperativer Komplikationen bzw. verlängerter Rekonvaleszenz nur ca. 30-34\% aller Patienten mit einem duktalen Pankreaskarzinom eine adjuvante Chemotherapie erhalten, wird zunehmend für das Pankreaskarzinom eine neoadjuvante Radiochemotherapie diskutiert, auch unter der Prämisse, höhere Raten von primären Ro-Resektionen zu erzielen. In den Studien von Snady et al. 2000 und Wilkowki et al. 2003 wurde eine Ro-Resektion in 2 bzw. in 60\% der Fälle erzielt. Die Morphologie der Tumorregression wurde dabei nicht beschrieben und nicht quantifiziert. In einer neueren Studie (Lind et al. 2007) wurden dagegen die Resektate in Großflächenschnitten aufgearbeitet, sodass eine gute Übersicht über Regressionsphänomene und eine Quantifizierung möglich ist. Die Analyse (J.L.) ergab bei 8 von 17 primär irresektablen Karzinomen eine Ro-Resektion. Drei Fälle wiesen weniger als 10\% vitale Tumorzellen auf, davon in 2 Fällen nur wenige vitale residuelle Tumorzellen; 10-50\% vitale Tumorresiduen fanden sich in 3 Fällen und keine Regression in 2 Fällen. Resistent erwiesen sich besonders intraduktale und Tu-
mor-Inselzell-Komplexe. In Analogie zu bereits etablierten Schemata wird empfohlen, ein 4-stufiges Regressionsgrading ( $\mathrm{o}=$ keine Regression oder $>50 \%$ vitaler Tumor; 1 = partielle Regression, $10-50 \%$ vitaler Tumor; $2=<10 \%$ vitaler Tumor; 3a = komplette Regression, wenige degenerierte Tumorzellen; $3 \mathrm{~b}=$ komplette Regression, kein Tumor) anzuwenden.

In kasuistischen Mitteilungen wurde auch eine komplette Tumorregression beschrieben, weswegen sie prinzipiell Bestandteil einer Klassifikation sein sollte. Grundsätzlich wird derzeit eine neoadjuvante (Radio-)Chemotherapie nach der aktuellen $\mathrm{S}_{3}$-Leitlinie nur in Studien empfohlen.

Anschließend wurden 23 Vorträge präsentiert. Im Folgenden sollen einige Beiträge kurz hervorgehoben werden:

In der Sektion „Oberer GI-Trakt“ berichtete die Gruppe um J. Rüschoff (Kassel) in Kooperation mit Arbeitsgruppen aus Bonn, Shanghai/China und Mexico-City/Mexico über die Evaluation des Her2-Status in Magenkarzinomen. Hier wurde ein modifiziertes Immun-ScoringSystem evaluiert. Dabei zeigte sich eine Her2-Positivitätsrate von $10,7 \%$ bei Verwendung des beim Mammakarzinom gebräuchlichen immunhistochemischen (IHC-)Scoring-Systems (davon 83\% Adenokarzinome vom intestinalen Typ). Die Konkordanzrate zwischen IHC und FISH betrug 93\% (7,4\% der Fälle FISH-positiv); jedoch zeigten 11 FISH-positive Fälle einen IHC-Score 2+ oder negativ (1+). Diese Ergebnisse belegen, dass eine starke (inkomplette) basolaterale und fokale $(<10 \%)$ Anfärbung in Biopsien als positiv $\left(3^{+}\right)$gewertet werden kann. Die Prävalenz der Her2-Positivität in fortgeschrittenen Magenkarzinomen beträgt mit dieser Evaluationsmethode 22\% (TOGA-Studie). Dies zeigt, dass das Her2-Immunscoring-System beim Magenkarzinom modifiziert werden sollte, um die optimale $\mathrm{Pa}$ tientenpopulation für die Anti-Her2-Therapie zu erfassen.

Im Kapitel „Unterer GI-Trakt“ demonstrierte F. Prall (Rostock) die Möglichkeiten der molekularen Klassifizierung kolorektaler Karzinome nach Jass (2007). Es wurde eine systematische Analyse an 130 Kolorektalkarzinomen (KRK) mittels verschiedener molekularpathologischer 
Methoden (Sequenzierung, MSI-, CIMPund LOH-Analysen) durchgeführt. Dabei zeigte sich, dass die KRK-Klasse 1 (sporadisch MSI-H: CIMP-H, MSI-H, MLH1+, B-raf+, CIN-; n=13), die KRK-Klasse 2 (sporadisch aus serratierten Läsionen ohne MSI-H: CIMP-L, B-raf+, MSS/MSI-L; $\mathrm{n}=4$ ) und die KRK-Klasse 5 (HNPCC: CIMP, MLH1-, MSI-H, B-raf-, APC-, p53-, CIN-; n=4) molekular gut abgegrenzt werden können. Zum klassischen FAP-Typ mit Adenom-Karzinom-Sequenz (KRK-Klasse 4; CIMP-, MSS/MSI-L, $\mathrm{CIN}+$ und/oder $\mathrm{p} 53+, \mathrm{APC}+, \mathrm{K}$-ras+) ließen sich 47 Tumoren zuordnen, plus wahrscheinlich weitere 24 Karzinome trotz CIN- aufgrund der übrigen Markerkonstellation; 13 Fälle erfüllten die Kriterien der "gemischten“ Klasse (KRK-Klasse 3: CIMP-L, MSS/MSI-L, K-ras+), jedoch blieb die Unterscheidung zwischen den KRK-Klassen 3 und 4 in einem beträchtlichen Anteil der Tumoren $(n=25)$ problematisch, wobei sich diese Tumoren keiner Klasse eindeutig zuordnen ließen.

Eine Wiener Gruppe um A. Soleiman konnte zeigen, dass das Protocadherin MUCDHL am Übergang vom proliferativen zum differenzierten Kryptenepithel exprimiert wird. Neoplastische Zellen exprimieren nur geringe MUCDHL-Level in kolorektalen Adenomen, während beim Übergang ins invasive Karzinom MUCDHL vollständig fehlt. Die Daten zeigen, dass die Bildung des MUCDHL/ $\beta$-Catenin-Komplexes eine bislang unbekannte Zellzyklusregulation in der kolorektalen Krypte via $\beta$-Catenin/TCF-Suppression triggert und so zur Differenzierung des kolorektalen Epithels beiträgt.

Eine Heidelberger Arbeitsgruppe (H. Bläker et al.) untersuchte den genetischen Hintergrund von Non-Polypose-, Non-HNPCC und nicht-CED-assoziierten Dünn- und Dickdarmkarzinomen bei jüngeren Patienten ( $\leq 35$ Jahre). Mittels einer Kombination von CGH, LOHund Genanalysen konnten sie in einer 35Mbp-Region auf Chromosom 6q (6q1422) die Existenz eines neuen 6q-assoziierten Krebs-Suszeptibilitäts-Syndroms postulieren.

Die auf dem Gebiet der humanen $\mathrm{He}$ patokarzinogenese sehr aktive Heidelberger Arbeitsgruppe um P. Schirmacher stellte die Ergebnisse umfangreicher geno- mischer Untersuchungen vor, die zeigen, dass es bei der Entstehung des humanen Leberzellkarzinoms ätiologieabhängige und -unabhängige molekulare Mechanismen gibt. So unterscheidet sich die Karzinogenese des kryptogenen HCC von der anderer ätiologischer Subgruppen zumindest im Hinblick auf einen 8q-Zugewinn und eine MYC-Aktivierung. Darüber hinaus repräsentiert die Aktivierung der Onkogene MDM 4 und eEF1A2 einen ätiologieunabhängigen Mechanismus, der vermutlich die $\mathrm{TP}_{53}$-abhängige Proliferationskontrolle in einem Teil der HCCs überwindet. Therapeutische Ansätze, die auf MDM 4 zielen, könnten so die bislang ungünstige Prognose der Patienten möglicherweise verbessern.

Vor dem gleichen Hintergrund stellte J.P. Theurillat (Zürich) die Ergebnisse der URI-Expression in HCCs vor; bei URI handelt es sich um ein ungewöhnlich großes Mitglied der Prefoldin-Familie, welche die Verfügbarkeit von Nährstoffen funktionell mit der Gentranskription verbindet. In einer TMA-Studie konnte gezeigt werden, dass die in $20 \%$ der Fälle immunhistologisch nachweisbare URI-Expression direkt mit dem Proliferationsindex und der Tumorgröße korreliert. Patienten mit URI-positiven HCCs zeigten überdies einen statistisch signifikant ungünstigeren Verlauf. Diese Daten sprechen dafür, dass URI ein neues Onkogen beim hepatozellulären Karzinom darstellt.

\section{Schwerpunkte der Tätigkeit der AG GI-Pathologie}

Die AG GI-Pathologie soll allen Mitgliedern der DGP, die sich für GI-Pathologie interessieren, ein wissenschaftliches Forum bieten. Die Mitgliedschaft steht allen Interessierten offen, es ist lediglich ein formloser Aufnahmeantrag an den Sprecher der AG erforderlich, der dann laut AG-Geschäftsordnung zusammen mit dem Beirat über die Aufnahme entscheidet. Da bislang kein schriftlich fixiertes Mitgliederverzeichnis existierte, wurden im Rahmen der diesjährigen AG-Sitzung Listen verteilt, aus denen prospektiv ein Verzeichnis erstellt werden soll, um die Kommunikation innerhalb der AG zu verbessern (so sollen künftig Informati- onen über die Arbeitsschwerpunkte und die Tätigkeit der AG auf der Homepage der DGP publiziert sowie die Leitlinien zu den GI-Tumoren und eine Übersicht zum Regressionsgrading zur Verfügung gestellt werden).

Die AG organisiert in Abstimmung mit dem Vorstand der DGP die jährlich im Rahmen der DGP-Sitzung stattfindende Sitzung einschließlich Mitgliederversammlung.

Weitere Aufgaben der AG bestehen darin, Sitzungen mit aktuellen Themen aus der GI-Pathologie für wissenschaftliche Tagungen der klinischen Fachgesellschaften auf dem GI-Sektor (z. B. DGVS, DGE-BV u. a.) zu organisieren. Dies kann in Form eigener Sitzungen erfolgen oder durch die Unterstützung der Veranstalter bei der Auswahl von Referenten. Schließlich werden auch Ringversuche und Konsensus-Konferenzen durchgeführt (zuletzt am 26. April 2008 in Düsseldorf zum Thema „Serratierte Läsionen im Kolorektum“; Koordination: D. Aust; Dresden - Publikation noch in diesem Jahr vorgesehen) sowie Leitlinien entwickelt (aktuell die S2Leitlinie zur Diagnose der alkoholischen und nicht-alkoholischen Steatohepatitis; Koordination: A. Tannapfel, Bochum).

Nach der Geschäftsordnung der AG werden von den Mitgliedern per Akklamation ein Sprecher und ein Beirat mit 4-jähriger Amtszeit bestimmt. In Berlin wurde G. Baretton, Dresden, für weitere 4 Jahre als Sprecher gewählt. Der Beirat wurde erweitert; ihm gehören an: D. Aust, Dresden, S. Baldus, Düsseldorf, I. Esposito, München, G. Faller, Karlsruhe, A. Jung, München, H. Koch, Freiburg, C. Langner, Graz, J. Lüttges, Saarbrücken, J. Rüschoff, Kassel, P. Schirmacher, Heidelberg, A. Tannapfel, Bochum, M. Vieth, Bayreuth, E. Wardelmann, Bonn, M. Werner, Freiburg. Der Beirat trifft sich außer im Rahmen der DGP-Tagung jährlich im Herbst.

\section{Korrespondenzadresse \\ Prof. Dr. G.B. Baretton}

Institut für Pathologie,

Universitätsklinikum Dresden

Fetscherstraße 74, 01307 Dresden

gustavo.baretton@uniklinikum-dresden.de

Interessenkonflikt. Der korrespondierende Autor gibt an, dass kein Interessenkonflikt besteht. 